\title{
Definition and Existence of Multichannel Scattering States
}

\author{
WERNER SANDHAS* \\ Institut für Theoretische Physik der Freien Universität Berlin \\ Received July 4, 1966
}

\begin{abstract}
The field theoretical formulation of quantum mechanics is used to consider the nonrelativistic multichannel scattering theory. With the help of appropriately constructed time dependent creation operators, Hilbert vectors are formed whose limits in time can be defined as multichannel scattering states in the usual sense. The existence of these states is proved under certain assumptions for the potential, by showing the convergence of the above mentioned operators. The commutation relations for the limits of these operators are given.
\end{abstract}

\section{Introduction}

Several formulations have been proposed to give a basis for the description of multichannel scattering processes. The first sufficiently general and correct of these approaches is due to Ekstein [1]. The essential point of his method is to define scattering states as limits of appropriately constructed time dependent Hilbert vectors. In contrast to other formulations channel Møller operators ${ }^{1}$ are not needed and therefore it is not necessary to split the total Hamiltonian into a channel Hamiltonian and the interactions between the fragments. In particular the formalism developed in Ref. [1] makes it possible to treat scattering processes of identical particles without disregarding the Pauli principle during parts of the calculation. Thus it can be transfered to field theory which automatically assures the Pauli principle. Furthermore, with this method scattering states can possibly be defined, even if channel Møller operators do not exist (a discussion of these questions is given in the review article of Brenig and HAag [3]).

Refering to EKsteIN's basic idea and generalizing them HAaG [4] has defined "in"-respectively "out"-states for the scattering of (in general composite) particles in the relativistic field theory. Furthermore he proved the existence of these asymptotic states. This proof was made rigorous by RUELLE [5] under the premises of the Garding-Wightman axioms and additional spectral conditions. Especially, RuELLE was able to show the

* Present address: Physikalisches Institut der Universität Bonn.

1 See, e.g., Ref. [2]. 
validity of the "spacelike asymptotic condition" decisive for HAAG's treatment. The physical and formal transparency, achieved in these papers, suggests the study of the analogous, but more simple, problem in nonrelativistic multichannel scattering theory, considering the procedures used in these papers.

In what follows Sec. 2 explains our notations and assumptions. Corresponding to the prescription of Ref. [4] in Sec. 3 multiparticle operators are constructed which upon acting on the vacuum state create bound states. In Sec. 4, using these operators, we form time dependent Hilbert vectors and define scattering states as their limits in time. The existence of these states is proved in Sec. 5, by showing first the convergence of the above mentioned multiparticle operators to asymptotic "in"- and "out"operators. The proof, which is related to the procedure used by Coor and HACK [6], [7] and discussed in Ref. [3] is done under two different assumptions for the potential (compare (5.13) and (5.20)). The commutation relations for the asymptotic operators are given in Sec. 6. Furthermore we show (Sec. 7) that the Hilbert spaces spanned by the scattering states are defined independently of the special choice of wave packets used for the construction.

The considerations carried out in the following are done with respect to the nonrelativistic multichannel scattering theory, but can also be regarded as an illustration of certain methods (mentioned above) in relativistic field theory by a familiar, physically completely interpretable theory. Of course, the differences in detail should not be overlooked.

\section{Assumptions and notations}

A system of identical bosons or fermions of mass $m$ will be treated. The $n$-particle Hamiltonian in configuration space representation is given by

$$
H^{(n)}\left(\mathbf{x}_{1}, \ldots, \mathbf{x}_{n}\right)=-\frac{1}{2 m} \sum_{i=1}^{n} \nabla_{\mathbf{x}_{i}}^{2}+\frac{1}{2} \sum_{i \neq j}^{n} V\left(\mathbf{x}_{i}-\mathbf{x}_{j}\right)
$$

where we take $\hbar=1$. For the (real) potential $V\left(\mathbf{x}_{i}-\mathbf{x}_{j}\right)$ we further require $V\left(\mathbf{x}_{i}-\mathbf{x}_{j}\right)=V\left(\mathbf{x}_{j}-\mathbf{x}_{i}\right)$.

Instead of this representation we shall use the field theoretical formulation $^{2}$ especially suited for multiparticle problems, i.e., field operators $\psi(\mathbf{x})$ are introduced which satisfy the commutation rules

$$
\begin{aligned}
{\left[\psi\left(\mathbf{x}^{\prime}\right), \psi^{+}(\mathbf{x})\right]_{\mp} } & =\delta\left(\mathbf{x}^{\prime}-\mathbf{x}\right), \\
{\left[\psi^{+}\left(\mathbf{x}^{\prime}\right), \psi^{+}(\mathbf{x})\right]_{\mp} } & =\left[\psi\left(\mathbf{x}^{\prime}\right), \psi(\mathbf{x})\right]_{\mp}=0 .
\end{aligned}
$$

${ }^{2}$ Concerning this method compare, e.g., Ref. [8], Chap. 6, where further literature is given. For applications of this formalism to nonrelativistic scattering theory, sce, e.g., Ref. [9]. 
In (2.2) the commutators hold in the boson case, the anticommutators in the fermion case. Here and in the following the variable $\mathbf{x}$ includes also the spin variable, if particles with spin are considered. In this sense the integration over $\mathbf{x}$ means simultaneously summation over the spin variable. With the help of the operators $\psi(\mathbf{x})$ the Hamiltonian, introduced by (2.1), is written in the form

$$
\begin{aligned}
H=\int d^{3} \mathbf{x} & \psi^{+}(\mathbf{x})\left(-\frac{1}{2 m} \nabla_{\mathbf{x}}^{2}\right) \psi(\mathbf{x})+ \\
& +\frac{1}{2} \int d^{3} \mathbf{x}^{\prime} \int d^{3} \mathbf{x} \psi^{+}\left(\mathbf{x}^{\prime}\right) \psi^{+}(\mathbf{x}) V\left(\mathbf{x}^{\prime}-\mathbf{x}\right) \psi(\mathbf{x}) \psi\left(\mathbf{x}^{\prime}\right) .
\end{aligned}
$$

This representation of $H$ is independent of the particle number $n$, thus it combines the operators $H^{(n)}$ for arbitrary $n$.

To fix the notations used in the following we summarize some further important relations. By applying the field operators $\psi^{+}\left(\mathbf{x}_{i}\right)$ on the vacuum $\Phi_{0}$ one gets the (anti-)symmetrical position states

$$
\Phi_{\mathbf{x}_{1}, \ldots, \mathbf{x}_{n}}=\frac{1}{\sqrt{n !}} \psi^{+}\left(\mathbf{x}_{1}\right) \ldots \psi^{+}\left(\mathbf{x}_{n}\right) \Phi_{0}
$$

which are (impoper) eigenvectors of the particle number operator

$$
N=\int d^{3} \mathbf{x} \psi^{+}(\mathbf{x}) \psi(\mathbf{x})
$$

and of the center-of-mass operator

$$
\mathbf{R}=\frac{\int d^{3} \mathbf{x} \psi^{+}(\mathbf{x}) \mathbf{x} \psi(\mathbf{x})}{N}
$$

belonging to the eigenvalues $n$ and $\frac{1}{n}\left(\mathbf{x}_{1}+\cdots+\mathbf{x}_{n}\right)$, respectively.

The total momentum operator has the form

$$
\mathbf{P}=\int d^{3} \mathbf{x} \psi^{+}(\mathbf{x}) \frac{\mathbf{1}}{i} \boldsymbol{\nabla}_{\mathbf{x}} \psi(\mathbf{x}),
$$

thus the energy operator of the center-of-mass motion is

By the transformation

$$
H-H^{\prime}=\frac{\mathbf{P}^{\mathbf{2}}}{2 N m} .
$$

$$
\psi(\mathbf{x}, t)=e^{i H t} \psi(\mathbf{x}) e^{-i H t}
$$

Heisenberg operators $\psi(\mathbf{x}, t)$ are associated to the Schrödinger operators $\psi(\mathbf{x})$ which satisfy equal-time commutation relations corresponding to (2.2).

We note that under the assumptions (5.13) or (5.20), required in the following for the potential, the Hamiltonian given by (2.1) or (2.3) is essentially self-adjoint if Hilbert spaces $\mathscr{H}$ with finite particle numbers are considered [10]. That is, the closure of $H$ is selfadjoint. In (2.9) and in the following $H$ shall designate this closure. Thus $e^{ \pm i H t}$ is uniquely defined in the whole Hilbert space $\mathscr{H}$. 
Finally the relation

$$
i \dot{\psi}(\mathbf{x}, t)=[\psi(\mathbf{x}, t), H]=\left(-\frac{1}{2 m} \nabla_{\mathbf{x}}^{2}+U(\mathbf{x}, t)\right) \psi(\mathbf{x}, t)
$$

with

$$
U(\mathbf{x}, t)=U^{+}(\mathbf{x}, t)=\int d^{3} \mathbf{x}^{\prime} \psi^{+}\left(\mathbf{x}^{\prime}, t\right) V\left(\mathbf{x}^{\prime}-\mathbf{x}\right) \psi\left(\mathbf{x}^{\prime}, t\right)
$$

should be mentioned.

\section{Multiparticle field operators}

For the description of multiparticle bound states we introduce $n$-particle field operators (compare [1], [4], [5])

$B_{n, v}(\mathbf{x}, t)$

$=\int d^{3} \mathbf{x}_{1} \ldots \int d^{3} \mathbf{x}_{n} g_{v}\left(\mathbf{x}-\mathbf{x}_{1}, \ldots, \mathbf{x}-\mathbf{x}_{n}\right) \frac{1}{\sqrt{n !}} \psi\left(\mathbf{x}_{n}, t\right) \ldots \psi\left(\mathbf{x}_{1}, t\right)$.

The functions $g_{v}\left(\mathbf{x}-\mathbf{x}_{1}, \ldots, \mathbf{x}-\mathbf{x}_{n}\right)$ shall now be restricted in the following way:

I. $\int d^{3} \mathbf{x} f(\mathbf{x}) g_{v}\left(\mathbf{x}-\mathbf{x}_{1}, \ldots, \mathbf{x}-\mathbf{x}_{n}\right)$ is square-integrable in $\mathbf{x}_{1}, \ldots, \mathbf{x}_{n}$, i.e. $\in L^{2}\left(R^{3 n}\right)$, for all test functions $f(\mathbf{x})$ of the class $\mathscr{S}[11]$.

II. $g_{v}\left(\mathbf{x}-\mathbf{x}_{1}, \ldots, \mathbf{x}-\mathbf{x}_{n}\right)$ is a symmetric function of the variables $\left(\mathbf{x}-\mathbf{x}_{i}\right)$ in the boson case, an antisymmetric function in the fermion case.

III. $g_{v}\left(\mathbf{x}-\mathrm{x}_{1}, \ldots, \mathrm{x}-\mathrm{x}_{n}\right)$ is chosen such that the state $B_{n, v}^{+}(\mathbf{x}, 0) \Phi_{0}$ describes a particle system with the center-of-mass $\mathbf{x}$, i.e., this state is an (improper) eigenvector of $\mathbf{R}$ to the eigenvalue $\mathbf{x}$ :

$$
\mathbf{R} B_{n, v}^{+}(\mathbf{x}, 0) \Phi_{0}=\mathbf{x} B_{n, v}^{+}(\mathbf{x}, 0) \Phi_{0} .
$$

From (3.2) it follows (by suitable normalization)

$\int g_{v}^{*}\left(\mathbf{x}^{\prime}-\mathbf{x}_{1}, \ldots, \mathbf{x}^{\prime}-\mathbf{x}_{n}\right) g_{\nu}\left(\mathbf{x}-\mathbf{x}_{1}, \ldots, \mathbf{x}-\mathbf{x}_{n}\right) \times$

$$
\times d^{3} \mathbf{x}_{1} \ldots d^{3} \mathbf{x}_{n}=\delta\left(\mathbf{x}^{\prime}-\mathbf{x}\right) .
$$

IV. Furthermore $g_{v}\left(\mathbf{x}-\mathbf{x}_{1}, \ldots, \mathbf{x}-\mathbf{x}_{n}\right)$ has the property that $B_{n, v}^{+}(\mathbf{x}, 0)$ operating on the vacuum creates a $n$-particle bound state of binding energy $E_{p}$. By (2.8) this property means ${ }^{3}$

$$
\left(H^{\prime}-E_{v}\right) B_{n, v}^{+}(\mathrm{x}, 0) \Phi_{0}=0 \text {. }
$$

For an alternative formulation of condition IV we regard a system of solutions of the free one-particle Schrödinger equation with mass $M=n \cdot m$ :

$$
f_{\alpha}(\mathbf{x}, t)=\frac{1}{(2 \pi)^{3 / 2}} \int d^{3} \mathbf{p} e^{i\left(\mathbf{p} \cdot \mathbf{x}-\frac{\mathbf{p}^{2}}{2 M} t\right)} f_{\alpha}(\mathbf{p}) .
$$

The $f_{\alpha}(\mathbf{p}) \in \mathscr{S}$ are chosen to form a complete orthonormal system in $L^{2}\left(R^{3}\right)$.

${ }^{3}$ The construction of the fields $B_{n, v}(\mathbf{x}, 0)$ can be called "solution of the oneparticle problem" [1], [4] if each $n$-particle bound state is called "one particle of mass $M=n \cdot m$ ".

25 Commun. math. Phys., Vol. 3 
Defining

we have

$$
b_{n \alpha \nu}(t)=\int d^{3} \mathbf{x} f_{\alpha}^{*}(\mathbf{x}, t) e^{i E_{\nu} t} B_{n, \nu}(\mathbf{x}, t)
$$

with

$$
b_{n \alpha \nu}^{+}(t) \Phi_{0}=\int d^{3} \mathbf{p} f_{\alpha}(\mathbf{p}) e^{i\left(H-\frac{\mathbf{p}^{2}}{2 M}-E_{\nu}\right) t} b_{n, v}^{+}(\mathbf{p}, 0) \Phi_{0}
$$

$$
b_{n, v}(\mathbf{p}, t)=\frac{1}{(2 \pi)^{3 / 2}} \int d^{3} \mathbf{x} e^{-i \mathbf{p} \cdot \mathbf{x}+i\left(\frac{\mathbf{p}^{2}}{2 M}+E_{v}\right) t} B_{n, v}(\mathbf{x}, t) .
$$

$b_{n, v}^{+}(\mathbf{p}, 0) \Phi_{0}$ is an (improper) eigenvector of the total momentum P. It therefore follows from (3.7)

$$
b_{n \alpha \nu}^{+}(t) \Phi_{0}=e^{i\left(H^{\prime}-E_{\nu) t}\right.} b_{n \alpha \nu}^{+}(0) \Phi_{0} .
$$

This implies that condition IV is equivalent to

Now we have

$$
\frac{d}{d t} b_{n \alpha \nu}^{+}(t) \Phi_{0}=0
$$

$$
\begin{gathered}
\frac{d}{d t} b_{n \alpha \nu}^{+}(t)=i \int d^{3} \mathbf{x} f_{\alpha}(\mathbf{x}, t) e^{-i E_{v} t} \int d^{3} \mathbf{x}_{1} \ldots \int d^{3} \mathbf{x}_{n} \times \\
\times\left\{\left[-E_{v}+\frac{1}{2 M} \nabla_{\mathbf{x}}^{2}-\frac{1}{2 m} \sum_{i} \nabla_{\mathbf{x}_{i}}^{2}+\sum_{i<j} V\left(\mathbf{x}_{i}-\mathbf{x}_{j}\right)\right] g_{\nu}^{*}\left(\mathbf{x}-\mathbf{x}_{1}, \ldots, \mathbf{x}-\mathbf{x}_{n}\right)\right\} \times \\
\times \frac{1}{\sqrt{n !}} \psi^{+}\left(\mathbf{x}_{1}, t\right) \ldots \psi^{+}\left(\mathbf{x}_{n}, t\right)+i \int d^{3} \mathbf{x} f_{\alpha}(\mathbf{x}, t) e^{-i E_{v} t} \int d^{3} \mathbf{x}_{1} \ldots \\
\ldots \int d^{3} \mathbf{x}_{n} g_{v}^{*}\left(\mathbf{x}-\mathbf{x}_{1}, \ldots, \mathbf{x}-\mathbf{x}_{n}\right) \frac{1}{\sqrt{n !}} \psi^{+}\left(\mathbf{x}_{1}, t\right) \ldots \psi^{+}\left(\mathbf{x}_{n}, t\right) \sum_{i} U\left(\mathbf{x}_{i}, t\right) .
\end{gathered}
$$

Eq. (3.10) gives, since $U\left(\mathbf{x}_{i}, t\right) \Phi_{0}=0$,

$$
\begin{aligned}
& {\left[-E_{v}+\frac{1}{2 M} \nabla_{\mathbf{x}}^{2}-\frac{1}{2 m} \sum_{i} \nabla_{\mathbf{x}_{i}}^{2}+\frac{1}{2} \sum_{i \neq j} V\left(\mathbf{x}_{i}-\mathbf{x}_{j}\right)\right] \times } \\
& \times g_{v}^{*}\left(\mathbf{x}-\mathbf{x}_{1}, \ldots, \mathbf{x}-\mathbf{x}_{n}\right)=0 .
\end{aligned}
$$

Thus (3.4) or (3.10) leads to the eigenvalue problem (3.12) which fixes (together with condition I, II, III) the binding energies $E_{v}$ and the corresponding eigenfunctions $g_{v}^{*}\left(\mathbf{x}-\mathbf{x}_{1}, \ldots, \mathbf{x}-\mathbf{x}_{n}\right)$, where $\nu$ contains all the quantum numbers necessary to characterize the eigenfunctions.

Eqs. (3.11) and (3.12) yield, with condition II,

$$
\begin{gathered}
\dot{b}_{n \alpha \nu}^{+}(t)=i \cdot n \int d^{3} \mathbf{x} f_{\alpha}(\mathbf{x}, t) e^{-i E_{\nu} t} \int d^{3} \mathbf{x}_{1} \ldots \int d^{3} \mathbf{x}_{n} g_{\nu}^{*}\left(\mathbf{x}-\mathbf{x}_{1}, \ldots, \mathbf{x}-\mathbf{x}_{n}\right) \times \\
\times \frac{1}{\sqrt{n !}} \psi^{+}\left(\mathbf{x}_{1}, t\right) \ldots \psi^{+}\left(\mathbf{x}_{n}, t\right) U\left(\mathbf{x}_{1}, t\right) .
\end{gathered}
$$

Finally we note the estimate important for subsequent use

$$
\left|f_{\alpha}(\mathbf{x}, t)\right| \leqq \frac{c_{\alpha}}{|t|^{3 / 2}}
$$


which can be proved immediately [12] if one considers that from (3.5) follows (for $t \neq 0$ ):

$$
f_{\alpha}(\mathbf{x}, t)=\left(\frac{M}{2 \pi i t}\right)^{3 / 2} \int d^{3} \mathbf{x}^{\prime} e^{i \frac{M}{2 t}\left(\mathbf{x}-\mathbf{x}^{\prime}\right)^{2}} f_{\alpha}\left(\mathbf{x}^{\prime}, 0\right)
$$

with $f_{\alpha}(\mathbf{x}, 0) \in \mathscr{S}$.

\section{Scattering states}

With the help of the creation operators $b_{n_{i} \alpha_{i} \nu_{i}}^{+}(t)$ we now construct a system of time dependent Hilbert vectors

$$
\Phi_{\eta}(t)=\Phi_{n_{1} \alpha_{1} \nu_{1}, \ldots, n_{k} \alpha_{k} \nu_{k}}^{(t)}=b_{n_{1} \alpha_{1} \nu_{1}}^{+}(t) \ldots b_{n_{k} \alpha_{k} \nu_{k}}^{+}(t) \Phi_{0}, \quad(k<\infty) .
$$

The strong limits of these vectors

$$
\Phi_{\eta}^{\text {out }}=\Phi_{n_{1} \alpha_{1} \nu_{1}, \ldots, n_{k} \alpha_{k} \nu_{k}}^{\text {out }}=\lim _{t \rightarrow \pm \infty} \Phi_{n_{1} \alpha_{1} \nu_{1}, \ldots, n_{k} \alpha_{k} \nu_{k}}^{(t)}
$$

are defined as scattering states, which are associated with certain initial ("in"-) or final ("out"-) configurations, characterized by the quantum numbers $\left(n_{1} \alpha_{1} v_{1}, \ldots, n_{k} \alpha_{k} v_{k}\right)=\eta$. The Hilbert space, i.e., the closed linear manifold spanned by the in- (out-) Hilbert vectors is called ${ }^{4}$ $\mathscr{H}^{\text {in }}\left(\mathscr{H}^{\text {out }}\right)$.

The definition of scattering states by (4.2) is meaningful and in accordance with the one given first by EKsTEIN [1] and discussed in the review article of BRENIG and HAAG [3]. This can be seen by writing (4.2) in the form

$$
\begin{aligned}
& \lim _{t \rightarrow \pm \infty}\left\|\Phi_{\eta}^{\mathrm{ex}}-\Phi_{\eta}(t)\right\|=\lim _{t \rightarrow \pm \infty} \| e^{-i H t} \Phi_{\eta}^{\mathrm{ex}}-\int d^{3} \mathbf{p}_{1} \ldots \int d^{3} \mathbf{p}_{k} \times \\
& \times f_{\alpha_{1}}\left(\mathbf{p}_{1}\right) \ldots f_{\alpha_{k}}\left(\mathbf{p}_{k}\right) e^{-i\left(\frac{\mathbf{p}_{1}^{2}}{2 M_{1}}+E_{\nu_{1}}+\cdots+\frac{\mathbf{p}_{k}^{2}}{2 M_{k}}+E_{\nu_{k}}\right) t} \Phi_{n_{1} \mathbf{p}_{1} \nu_{1}, \ldots, n_{k} \mathbf{p}_{k} \nu_{k} \|}=0
\end{aligned}
$$

where $b_{n_{1}, v_{1}}^{+}\left(\mathbf{p}_{1}, 0\right) \ldots b_{n_{k}, v_{k}}^{+}\left(\mathbf{p}_{k}, 0\right) \Phi_{0}=\Phi_{n_{1} \mathbf{p}_{1} \nu_{1}, \ldots, n_{k} \mathbf{p}_{k} v_{k}}$ has been introduced. Eq. (4.3) shows that for sufficiently large positive or negative times the Schrödinger states $e^{-i H t} \Phi_{\eta}^{\text {ex }}$ differ "arbitrarily little" from a wave packet of free moving (generally composite) particles, characterized by the quantum numbers $\eta=\left(n_{1} \alpha_{1} \nu_{1}, \ldots, n_{k} \alpha_{k} v_{k}\right)$. It should be noted that $\mathscr{H}^{\mathrm{ex}}$ according to the definition (4.2) contains besides the scattering states in the proper sense $(k>1)$ also the bound-states $\Phi_{n \alpha \nu}^{\mathrm{ex}}=b_{n \alpha \nu}^{+}(t) \Phi_{0}$ $=b_{n \alpha \nu}^{+}(0) \Phi_{0}$.

Because of (2.2) we have

$$
\left[b_{n_{i} \alpha_{i} v_{i}}^{+}(t), b_{n_{j} \alpha_{j} v_{j}}^{+}(t)\right]_{\mp}=0
$$

where the anticommutator holds in the fermion case if $n_{i}$ as well as $n_{j}$ is odd, and the commutator in all other cases. Correspondingly the states $\Phi_{\eta}^{\text {ex }}$ are (anti-) symmetric with respect to an interchange of the sets of indices $\left(n_{i} \alpha_{i} v_{i}\right)$ and $\left(n_{j} \alpha_{j} \nu_{j}\right)$.

${ }^{4}$ We often use the designation "ex" for either "in" or "out". 


\section{Proof of existence}

To prove the existence of the scattering states defined by (4.2) we have to show the (strong) convergence of the Hilbert vectors (4.1). Instead of doing this directly ${ }^{5}$ we first prove that under certain assumptions for the potential the operators $b_{n \alpha \nu}^{+}(t)$ converge on all vectors $\Phi$ of the Hilbert space $\mathscr{H}_{N}$ if $\mathscr{H}_{N}$ contains only finite particle numbers $n \leqq N<\infty$. That is, we show that the norm

$$
\left\|\left(b_{n \alpha \nu}^{+}\left(t_{2}\right)-b_{n \alpha \nu}^{+}\left(t_{1}\right)\right) \Phi\right\|
$$

becomes arbitrarily small for sufficiently large $\left|t_{1}\right|$ and either $t_{2}>t_{1}>0$ or $t_{2}<t_{1}<0$. This norm can be written in the form

$$
\left\|\left(b_{n \alpha \nu}^{+}\left(t_{2}\right)-b_{n \alpha \nu}^{+}\left(t_{1}\right)\right) \Phi\right\|=\left\|\int_{t_{1}}^{t_{2}} d t \dot{b}_{n \alpha \nu}^{+}(t) \Phi\right\|
$$

which holds, because the integrand exists and depends continuously on $t$ in the norm topology ${ }^{6}$. The immediate consequence of $(5.1)$ is

$$
\left\|\left(b_{n \alpha \nu}^{+}\left(t_{2}\right)-b_{n \alpha \nu}^{+}\left(t_{1}\right)\right) \Phi\right\| \leqq\left|\int_{t_{1}}^{t_{2}} d t\left\|\dot{b}_{n \alpha \nu}^{+}(t) \Phi\right\|\right|
$$

which leads to the sufficient condition for the strong convergence of $b_{n \alpha \nu}^{+}(t)$ :

$$
\left\|\dot{b}_{n \alpha v}^{+}(t) \Phi\right\| \leqq \text { const } \cdot|t|^{-1-\gamma}, \quad \gamma>0 .
$$

To prove this estimate we regard the vectors

$$
\Phi_{F}^{(j)}=\int d^{3} \mathbf{y}_{1} \ldots \int d^{3} \mathbf{y}_{j} F\left(\mathrm{y}_{1}, \ldots, \mathrm{y}_{j}\right) \frac{1}{\sqrt{j !}} \psi^{+}\left(\mathrm{y}_{1}\right) \ldots \psi^{+}\left(\mathrm{y}_{j}\right) \Phi_{0}
$$

of the Hilbert space $\mathscr{H}^{(j)}$ with fixed particle number $j$. The functions $F\left(\mathrm{y}_{1}, \ldots, \mathrm{y}_{j}\right) \in L^{2}\left(R^{3 j}\right)$ are chosen to be (anti-)symmetric in the (fermion-) boson case. This yields

$$
\left\|\Phi_{F^{\prime}}^{(j)}\right\|^{2}=\int d^{3} \mathbf{y}_{1} \ldots \int d^{3} \mathbf{y}_{j}\left|F\left(\mathbf{y}_{1}, \ldots, \mathbf{y}_{j}\right)\right|^{2} .
$$

Defining the operators

$$
\dot{b}_{n \alpha \nu}^{+}(t, 0)=e^{-i H t} \dot{b}_{n \alpha \nu}^{+}(t) e^{i H t}
$$

which are given explicitly by (3.13) having replaced there $\psi^{+}\left(\mathbf{x}_{i}, t\right)$ and $U\left(\mathbf{x}_{1}, t\right)$ by $\psi^{+}\left(\mathbf{x}_{i}\right)$ and $U\left(\mathbf{x}_{1}, 0\right)$, respectively, we get

$\dot{b}_{n \alpha \nu}^{+}(t, 0) \Phi_{F}^{(j)}=i \cdot n \cdot j \int d^{3} \mathbf{x}_{1} \ldots d^{3} \mathbf{x}_{n} \int d^{3} \mathbf{y}_{1} \ldots d^{3} \mathbf{y}_{j} \int \times$

$\times d^{3} \mathbf{x} f_{\alpha}(\mathbf{x}, t) g_{v}^{*}\left(\mathbf{x}-\mathbf{x}_{1}, \ldots, \mathbf{x}-\mathbf{x}_{n}\right) \times$

$\times V\left(\mathbf{x}_{1}-\mathbf{y}_{1}\right) F\left(\mathbf{y}_{1}, \ldots, \mathbf{y}_{j}\right) \frac{1}{\sqrt{n !}} \frac{1}{\sqrt{j !}} \psi^{+}\left(\mathbf{x}_{1}\right) \ldots \psi^{+}\left(\mathbf{x}_{n}\right) \psi^{+}\left(\mathbf{y}_{1}\right) \ldots \psi^{+}\left(\mathbf{y}_{j}\right) \Phi_{0}$.

5 A direct proof is given in Appendix C.

6 The continuous $t$-dependence follows from similar considerations used below for the estimate of (5.1) if the potential fulfils (5.13) or (5.20). 
With the help of the inequality

$$
\begin{aligned}
\| \int d^{3} \mathbf{x}_{1} \ldots \int d^{3} \mathbf{x}_{k} v\left(\mathbf{x}_{1}, \ldots,\right. & \left.\mathbf{x}_{k}\right) \psi^{+}\left(\mathbf{x}_{1}\right) \ldots \psi^{+}\left(\mathbf{x}_{k}\right) \Phi_{0} \|^{2} \leqq \\
& \leqq k ! \int d^{3} \mathbf{x}_{1} \ldots \int d^{3} \mathbf{x}_{k}\left|v\left(\mathbf{x}_{1}, \ldots, \mathbf{x}_{k}\right)\right|^{2}
\end{aligned}
$$

following from ScHWARz' inequality for functions $v\left(\mathbf{x}_{1}, \ldots, \mathbf{x}_{k}\right) \in L^{2}\left(R^{3 k}\right)$, one immediately has

$$
\begin{aligned}
\| \dot{b}_{n \alpha \nu}^{+}(t, 0) & \Phi_{F}^{(j)} \|^{2} \leqq K^{(n, j)} \int d^{3} \mathbf{x}_{1} \ldots d^{3} \mathbf{x}_{n} \int d^{3} \mathbf{y}_{1} \ldots d^{3} \mathbf{y}_{j} \times \\
& \times \int d^{3} \mathbf{x}^{\prime} f_{\alpha}^{*}\left(\mathbf{x}^{\prime}, t\right) g_{v}\left(\mathbf{x}^{\prime}-\mathbf{x}_{1}, \ldots, \mathbf{x}^{\prime}-\mathbf{x}_{n}\right) \int d^{3} \mathbf{x} f_{\alpha}(\mathbf{x}, t) \times \\
& \times g_{v}^{*}\left(\mathbf{x}-\mathbf{x}_{1}, \ldots, \mathbf{x}-\mathbf{x}_{n}\right) V^{2}\left(\mathbf{x}_{1}-\mathbf{y}_{1}\right)\left|F\left(\mathbf{y}_{1}, \ldots, \mathbf{y}_{j}\right)\right|^{2} \\
& =K^{(n, j)} \int d^{3} \mathbf{x}^{\prime} \int d^{3} \mathbf{x} \int d^{3} \mathbf{r} \int d^{3} \mathbf{y}_{1} \ldots d^{3} \mathbf{y}_{j} \times \\
& \times f_{\alpha}^{*}\left(\mathbf{x}^{\prime}+\mathbf{r}+\mathbf{y}_{1}, t\right) f_{\alpha}\left(\mathbf{x}+\mathbf{r}+\mathbf{y}_{1}, t\right) G\left(\mathbf{x}^{\prime}, \mathbf{x}\right) V^{2}(\mathbf{r})\left|F\left(\mathbf{y}_{1}, \ldots, \mathbf{y}_{j}\right)\right|^{2}
\end{aligned}
$$

Here the abbreviations $K^{(n, j)}=\frac{n \cdot j}{(n-1) !(j-1) !}(n+j) !, \mathbf{x}_{1}-\mathbf{y}_{1}=\mathbf{r}$, $(|\mathbf{r}|=r)$ and

$$
\begin{aligned}
G\left(\mathbf{x}^{\prime}, \mathbf{x}\right)=\int d^{3} \mathbf{x}_{2} \ldots \int d^{3} \mathbf{x}_{n} g_{v}\left(\mathbf{x}^{\prime}, \mathbf{x}^{\prime}-\right. & \left.\mathbf{x}_{2}, \ldots, \mathbf{x}^{\prime}-\mathbf{x}_{n}\right) \times \\
& \times g_{v}^{*}\left(\mathbf{x}, \mathbf{x}-\mathbf{x}_{2}, \ldots, \mathbf{x}-\mathbf{x}_{n}\right)
\end{aligned}
$$

have been introduced. The special properties of the functions $g_{v}$, shown, e.g., in Eq. (A.2), imply

$$
G\left(\mathbf{x}^{\prime}, \mathbf{x}\right)=\delta\left(\mathbf{x}^{\prime}-\mathbf{x}\right) G(\mathbf{x})
$$

where, as a consequence of condition $I, G(\mathbf{x})$ is an integrable function for $n \neq 1$ and equals $\delta(\mathbf{x})$ for $n=1^{7}$. Thus the inequality

$$
\begin{aligned}
\left\|\dot{b}_{n \alpha \nu}^{+}(t, 0) \Phi_{F}^{(j)}\right\|^{2} \leqq & K^{(n, j)} \int d^{3} \mathbf{x} \int d^{3} \mathbf{r} \int d^{3} \mathbf{y}_{1} \ldots d^{3} \mathbf{y}_{j} \times \\
& \times\left|f_{\alpha}\left(\mathbf{x}+\mathbf{r}+\mathbf{y}_{1}, t\right)\right|^{2} G(\mathbf{x}) V^{2}(\mathbf{r})\left|F\left(\mathbf{y}_{1}, \ldots, \mathbf{y}_{j}\right)\right|^{2}
\end{aligned}
$$

holds, which is essential for this proof.

If $V(\mathbf{r})$ satisfies the condition [6]

$$
\int V^{2}(\mathbf{r}) d^{3} \mathbf{r}<\infty
$$

Eq. (5.12) yields, under consideration of (3.14), the estimate

$$
\begin{aligned}
\left\|\dot{b}_{n \alpha \nu}^{+}(t, 0) \Phi_{F}^{(j)}\right\|^{2} \leqq K^{(n, j)} \frac{c_{\alpha}^{2}}{|t|^{3}} \int G(\mathbf{x}) d^{3} \mathbf{x} \int V^{2}(\mathbf{r}) d^{3} \mathbf{r} & \left\|\Phi_{F}^{(j)}\right\|^{2} \\
& =\frac{c_{\alpha, j}^{2}}{|t|^{3}}\left\|\Phi_{F}^{(j)}\right\|^{2}
\end{aligned}
$$

Here $c_{\alpha, j}$ is independent of $F$, hence $\dot{b}_{n \alpha \nu}^{+}(t, 0)$ is a bounded operator on $\mathscr{H}^{(j)}$. Considering (5.6) it follows from (5.14)

$$
\left\|\dot{b}_{n \alpha \nu}^{+}(t) \Phi_{F}^{(j)}\right\| \leqq \frac{c_{\alpha, j}}{|t|^{3 / 2}}\left\|e^{-i H t} \Phi_{F}^{(j)}\right\|=\frac{c_{\alpha, j}}{|t|^{3 / 2}}\left\|\Phi_{F}^{(j)}\right\|,
$$

7 The proof is given in Appendix A (see Eqs. (A.7)-(A.9)). 
i.e., the sufficient condition (5.3) given above for the convergence of $b_{n \alpha \nu}^{+}(t)$ is fulfilled. Therefore $b_{n \alpha v}^{+}(t)$ converges on $\mathscr{H}^{(j)}$ and thus on every Hilbert space $\mathscr{H}_{N}=\sum_{j=0}^{N} \oplus \mathscr{H}^{(j)}$ with $N<\infty$. We should stress that Eq. (5.15) implies not only strong but even uniform operator convergence on $\mathscr{H}_{N}{ }^{8}$.

The convergence of the states (4.1), i.e., the validity of (4.2) follows immediately from the strong convergence of the operators $b_{n \alpha \nu}^{+}(t)$ and of their boundedness with a bound independent of $t$. To show this boundedness we define, similarly to (5.6), the operator

and get, with (5.8),

$$
b_{n \alpha \nu}^{+}(t, 0)=e^{-i H t} b_{n \alpha \nu}^{+}(t) e^{i H t}
$$

$$
\left\|b_{n \alpha \nu}^{+}(t, 0) \Phi_{F}^{(j)}\right\|^{2} \leqq \frac{1}{n !} \frac{1}{j !}(n+j) !\left\|\Phi_{F}^{(j)}\right\|^{2} .
$$

The same estimate holds, of course, if $b_{n \alpha v}^{+}(t, 0)$ is replaced by $b_{n \alpha v}^{+}(t)$. This completes our proof.

With the definition

$$
b_{n \alpha \nu}^{+\stackrel{\text { out }}{n}=}=b_{n \alpha \nu}^{+ \text {ex }}=\lim _{t \rightarrow \pm \infty} b_{n \alpha \nu}^{+}(t)
$$

the scattering states (4.2) can now be written in the form

$$
\Phi_{n_{1} \alpha_{1} \nu_{1}, \ldots, n_{k} \alpha_{k} \nu_{k}}^{\mathrm{ex}}=b_{n_{1} \alpha_{1} \nu_{1}}^{+\mathrm{ex}} \ldots b_{n_{k} \alpha_{k} \nu_{k}}^{+\mathrm{ex}} \Phi_{0} .
$$

The existence of these states can also be proved under the following assumption for the potential [7]

$$
\begin{aligned}
& \iint_{r=0}^{r=r_{0}} V^{2}(\mathbf{r}) d^{3} \mathbf{r}<\infty \quad \text { for arbitrary but finite } r_{0} \\
& |V(\mathbf{r})| \leqq \frac{c}{r^{1+\beta}}, \quad \beta>0, \quad \text { for } r>r_{1}\left(r_{1}>0, \text { fixed }\right) .
\end{aligned}
$$

Using similar considerations as above we show in Appendix B, that

$$
\left\|\dot{b}_{n \alpha \nu}^{+}(t) \Phi^{(j)}\right\| \leqq \frac{c_{\alpha, j}}{|t|^{\mid+\gamma}}\left\|\Phi^{(j)}\right\|, \quad c_{\alpha, j}=c_{\alpha, j}\left(t_{0}\right),
$$

holds, with $\gamma>0$, if only $|t| \geqq t_{0}>0$. Thus (5.3) is fulfilled, i.e., the operators $b_{n \alpha \nu}^{+}(t)$ and therefore the states (4.1) converge.

Finally we note that from the boundedness of $b_{n \alpha \nu}^{+}(t)$ on $\mathscr{H}_{N}$ one can conclude that $b_{n \alpha \nu}(t)$ is also a bounded operator on $\mathscr{H}_{N}$ with $t$-independent bound. Furthermore, from the estimates (5.15) or (5.21) the convergence of $b_{n \alpha \nu}(t)$ can be derived. If, e.g., (5.21) is valid, Eq. (5.2) yields

$$
\left\|\left(b_{n \alpha \nu}^{+}\left(t_{2}\right)-b_{n \alpha \nu}^{+}\left(t_{1}\right)\right) \Phi^{(j)}\right\| \leqq \frac{c_{\alpha, j}^{\prime}}{\left|t_{1}\right|^{\gamma}}\left\|\Phi^{(j)}\right\|, \quad \gamma>0 .
$$

8 The norm defining the uniform operator topology depends on the particle number of $\mathscr{H}_{N}$. 
It hence follows

$\left\|\left(b_{n \alpha \nu}\left(t_{2}\right)-b_{n \alpha \nu}\left(t_{1}\right)\right) \Phi^{(j)}\right\|^{2} \leqq\left\|\Phi^{(j)}\right\| \frac{c_{\alpha, j-n}^{\prime}}{\left|t_{1}\right|^{\gamma}}\left\|\left(b_{n \alpha \nu}\left(t_{2}\right)-b_{n \alpha \nu}\left(t_{1}\right)\right) \Phi^{(j)}\right\|$

and therefore

$$
\left\|\left(b_{n \alpha \nu}\left(t_{2}\right)-b_{n \alpha \nu}\left(t_{1}\right)\right) \Phi^{(j)}\right\| \leqq \frac{c_{\alpha, j-n}^{\prime}}{\left|t_{1}\right|^{\gamma}}\left\|\Phi^{(j)}\right\|
$$

(for $n>j$ we may set $c_{\alpha, j-n}^{\prime}=0$ ). As the right-hand side of this inequality becomes arbitrarily small for sufficiently large $\left|t_{1}\right|$ the convergence of $b_{n \alpha \nu}(t)$ is proved on $\mathscr{H}^{(j)}$ and thus on the Hilbert space $\mathscr{H}_{N}$.

This justifies the definition analogous to (5.18)

$$
b_{n \alpha \nu}^{\text {in }}=b_{n \alpha \nu}^{\mathrm{ex}}=\lim _{t \rightarrow \pm \infty} b_{n \alpha \nu}(t) .
$$

\section{Commutation relations}

The convergence and boundedness of $b_{n \alpha \nu}^{+}(t)$ and $b_{n \alpha \nu}(t)$ yield

$$
\left[b_{n \alpha v}^{\text {out }} \stackrel{\text { out }}{\text { on }}, b_{n^{\prime} \alpha^{\prime} v^{\prime}}^{\text {in }}\right]_{\mp}=\left[b_{n \alpha \nu}^{+ \text {out }}, \stackrel{\text { in }}{\text { out }}, b_{n^{\prime} \alpha^{\prime} v^{\prime}}^{+ \text {in }}\right]_{\mp}=0 .
$$

Furthermore, defining

we have

$$
\left[b_{n \alpha \nu}(t), b_{n^{\prime} \alpha^{\prime} v^{\prime}}^{+}(t)\right]_{\mp}=\delta_{n n^{\prime}} \delta_{\alpha \alpha^{\prime}} \delta_{v v^{\prime}}+d_{n \alpha \nu, n^{\prime} \alpha^{\prime} y^{\prime}}^{(t)}
$$

$$
\left[b_{n \alpha v}^{\text {in }}, b_{n^{\prime} \alpha^{\prime} v^{\prime}}^{+ \text {in }}\right]_{\mp}^{\text {out }}=\delta_{n n^{\prime}} \delta_{\alpha \alpha^{\prime}} \delta_{v v^{\prime}}+\lim _{t \rightarrow \pm \infty} d_{n \alpha v, n^{\prime} \alpha^{\prime} v^{\prime}}^{(t)}
$$

We show that

which implies

$$
\lim _{t \rightarrow \pm \infty} d_{n \alpha v^{\prime}, n^{\prime} \alpha^{\prime} v^{\prime}}^{(t)}=0
$$

$$
\left[b_{n \alpha \nu}^{\text {in }}, \stackrel{\text { out }}{\text { out }}+b_{n^{\prime} \alpha^{\prime} \nu^{\prime}}^{\text {in }}\right]_{\mp}=\delta_{n n^{\prime}} \delta_{\alpha \alpha^{\prime}} \delta_{v v^{\prime}} .
$$

First we note that $d_{n \alpha v, n^{\prime} \alpha^{\prime} v^{\prime}}^{(t)}$ equals zero for $n=n^{\prime}=1$, otherwise it is a sum of terms

$$
\begin{array}{r}
\hat{d}(t)=\text { const } \int d^{3} \mathbf{x} \int d^{3} \mathbf{x}^{\prime} \int d^{3} \mathbf{x}_{1} \ldots d^{3} \mathbf{x}_{n} \int d^{3} \mathbf{x}_{i+1}^{\prime} \ldots d^{3} \mathbf{x}_{n^{\prime}}^{\prime} \times \\
\times f_{\alpha}^{*}(\mathbf{x}, t) g_{v}\left(\mathbf{x}-\mathbf{x}_{1}, \ldots, \mathbf{x}-\mathbf{x}_{i}, \mathbf{x}-\mathbf{x}_{i+1}, \ldots, \mathbf{x}-\mathbf{x}_{n}\right) \times \\
\times f_{\alpha^{\prime}}\left(\mathbf{x}^{\prime}, t\right) g_{v^{\prime}}^{*}\left(\mathbf{x}^{\prime}-\mathbf{x}_{1}, \ldots, \mathbf{x}^{\prime}-\mathbf{x}_{i}, \mathbf{x}^{\prime}-\mathbf{x}_{i+1}^{\prime}, \ldots, \mathbf{x}^{\prime}-\mathbf{x}_{n^{\prime}}^{\prime}\right) \times \\
\times \psi^{+}\left(\mathbf{x}_{i+1}^{\prime}, t\right) \ldots \psi^{+}\left(\mathbf{x}_{n^{\prime}}^{\prime}, t\right) \psi\left(\mathbf{x}_{i+1}, t\right) \ldots \psi\left(\mathbf{x}_{n}, t\right)
\end{array}
$$

where in any case $i \neq 0$ holds and for $n=n^{\prime}$ in addition $i \neq n$.

We now define, analogously to (5.6) and 5.16),

$$
\hat{d}(t, 0)=e^{-i H t} \hat{d}(t) e^{i H t}
$$


and get, with the help of (5.8),

$$
\begin{aligned}
& \left\|\hat{d}(t, 0) \Phi_{F}^{(j)}\right\|^{4} \leqq \text { const }\left\|\Phi_{F}^{(j)}\right\|^{4} \int d^{3} \mathbf{x}_{i+1} \ldots d^{3} \mathbf{x}_{n} \int d^{3} \mathbf{y}_{i+1} \ldots d^{3} \mathbf{y}_{n} \times \\
& \quad \times\left\{\mid \int d^{3} \mathbf{x}_{1} \ldots d^{3} \mathbf{x}_{i} \int d^{3} \mathbf{y}_{1} \ldots d^{3} \mathbf{y}_{i} \int d^{3} \mathbf{x}_{i+1}^{\prime} \ldots d^{3} \mathbf{x}_{n^{\prime}}^{\prime} \times\right. \\
& \quad \times \int d^{3} \mathbf{x} f_{\alpha}^{*}(\mathbf{x}, t) g_{v}\left(\mathbf{x}-\mathbf{x}_{1}, \ldots, \mathbf{x}-\mathbf{x}_{n}\right) \times \\
& \quad \times \int d^{3} \mathbf{x}^{\prime} f_{\alpha^{\prime}}\left(\mathbf{x}^{\prime}, t\right) g_{v^{\prime}}^{*}\left(\mathbf{x}^{\prime}-\mathbf{x}_{1}, \ldots, \mathbf{x}^{\prime}-\mathbf{x}_{i}, \mathbf{x}^{\prime}-\mathbf{x}_{i+1}^{\prime}, \ldots, \mathbf{x}^{\prime}-\mathbf{x}_{n^{\prime}}^{\prime}\right) \times \\
& \quad \times \int d^{3} \mathbf{y} f_{\alpha}(\mathbf{y}, t) g_{v}^{*}\left(\mathbf{y}-\mathbf{y}_{1}, \ldots, \mathbf{y}-\mathbf{y}_{n}\right) \times \\
& \left.\quad \times\left.\int d^{3} \mathbf{y}^{\prime} f_{\alpha^{\prime}}^{*}\left(\mathbf{y}^{\prime}, t\right) g_{v^{\prime}}\left(\mathbf{y}^{\prime}-\mathbf{y}_{1}, \ldots, \mathbf{y}^{\prime}-\mathbf{y}_{i}, \mathbf{y}^{\prime}-\mathbf{x}_{i+1}^{\prime}, \ldots, \mathbf{y}^{\prime}-\mathbf{x}_{n^{\prime}}^{\prime}\right)\right|^{2}\right\} .
\end{aligned}
$$

Under consideration of (3.14) the estimate

$$
\left\|\hat{d}(t, 0) \Phi_{F}^{(j)}\right\|^{4} \leqq \frac{c_{j}}{t^{6}}\left\|\Phi_{F}^{(j)}\right\|^{4}
$$

follows, which can be obtained by manipulations similar to those used in Sec. 5. However, not the behaviour of the potential, but the strong decrease (for large $\left|\xi_{i}\right|$ ) of the bound-state functions ${ }^{9} h_{v}\left(\xi_{1}, \ldots, \xi_{n-1}\right.$ ) is important in this case. For the validity of (6.9) it is already sufficient, that $\left|h_{v}\left(\xi_{1}, \ldots, \xi_{n-1}\right)\right|$ can be estimated by const $\cdot \prod_{i=1}^{n-1}\left|\xi_{i}\right|^{-3-\varepsilon}, \varepsilon>0$, for large $\left|\boldsymbol{\xi}_{i}\right|$.

In (6.9) the operator $\hat{d}(t, 0)$ can be replaced by $\hat{d}(t)$ and therefore our statement (6.4) is proved. It should be noted that the commutation relation (6.5) together with

$$
b_{n \alpha \nu}^{\mathrm{ex}} \Phi_{0}=\lim _{t \rightarrow \pm \infty} b_{n \alpha \nu}(t) \Phi_{0}=0
$$

implies the orthogonality of "in"- resp. "out"-states with non-identical sets of quantum numbers $\left(n_{1} \alpha_{1} \nu_{1}, \ldots, n_{k} \alpha_{k} v_{k}\right)$.

\section{Uniqueness of $\mathscr{H}^{\text {in }}$}

For the construction of the operators $b_{n \alpha \nu}^{+}(t)$ a special complete orthonormal system of functions $\tilde{f}_{\alpha}(\mathbf{p}) \in \mathscr{S}$ has to be chosen. Starting from a different set of functions $\tilde{f}_{\alpha}(\mathbf{p}) \in \mathscr{S}$, we can introduce the corresponding operators $\underline{b}_{n \alpha \nu}^{+}(t)$ which lead to operators $\underline{b}_{n \alpha \nu}^{+ \text {ex }}$ and states $\underline{\Phi}_{\eta}^{\text {ex }}$ as it was described in the previous sections.

From the completeness of the $f_{\alpha}(p)$ we now have

$$
\underline{f}_{\alpha}(\mathbf{x}, t)=\sum_{\alpha^{\prime}=1}^{\infty} c_{\alpha \alpha^{\prime}} f_{\alpha^{\prime}}(\mathbf{x}, t)
$$

where $\underline{f}_{\alpha}(\mathbf{x}, t)$ is given by (3.5), with $\tilde{f}_{\alpha}(\mathbf{p})$ instead of $\tilde{f}_{\alpha}(\mathbf{p})$. As a con-

${ }^{2}$ These functions and the variables $\xi_{i}$ are defined in Appendix A. 
sequence of Eq. (7.1) the right-hand side of the inequality

$$
\begin{aligned}
&\left\|\left(\underline{b}_{n \alpha \nu}^{+}(t)-\sum_{\alpha^{\prime}=1}^{a} c_{\alpha \alpha^{\prime}} b_{n \alpha^{\prime} v}^{+}(t)\right) \Phi_{F^{\prime}}^{(j)}\right\|^{2} \leqq \\
& \leqq \text { const } \int\left|\underline{f}_{\alpha}(\mathbf{x}, 0)-\sum_{\alpha^{\prime}=1}^{a} c_{\alpha \alpha^{\prime}} f_{\alpha^{\prime}}(\mathbf{x}, 0)\right|^{2} d^{3} \mathbf{x}\left\|e^{-i H t} \Phi_{F^{(j)}}^{(j)}\right\|^{2}
\end{aligned}
$$

becomes arbitrarily small for sufficiently large values of $a^{10}$.

Hence

and therefore

$$
\underline{b}_{n \alpha \nu}^{+\mathrm{ex}}=\sum_{\alpha^{\prime}=1}^{\infty} c_{\alpha \alpha^{\prime}} b_{n \alpha^{\prime} \nu}^{+\mathrm{ex}}
$$

$$
\Phi_{n_{1} \alpha_{1} \nu_{1}, \ldots, n_{k} \alpha_{k} \nu_{k}}^{\mathrm{ex}}=\sum_{\alpha_{1}^{\prime} \ldots \alpha_{k}}^{\sum} c_{\alpha_{1} \alpha_{1}^{\prime}} \ldots c_{\alpha_{k} \alpha_{k}^{\prime}} \Phi_{n_{1} \alpha_{1}^{\prime} \nu_{1}, \ldots, n_{k} \alpha_{k}^{\prime} \nu_{k}}^{\mathrm{ex}}
$$

is valid, which shows that $\mathscr{H}^{\text {in }}$ and $\mathscr{H}^{\text {out }}$ are defined uniquely, i.e., independently of the particular choice of the functions $f_{\alpha}(\mathbf{p})$.

Thus we may introduce distribution valued operators $b_{n, v}^{+ \text {ex }}(\mathbf{p})$ with

and

$$
b_{n \alpha \nu}^{+\mathrm{ex}}=\int d^{3} \mathbf{p} f_{\alpha}(\mathbf{p}) b_{n, \nu}^{+\mathrm{ex}}(\mathbf{p})
$$

$$
\underline{b}_{n \alpha \nu}^{+\mathrm{ex}}=\int d^{3} \mathbf{p} \underline{\tilde{f}}_{\alpha}(\mathbf{p}) b_{n, v}^{+\mathrm{ex}}(\mathbf{p}) \text {. }
$$

Now it can easily be seen, that $b_{n_{1}, \nu_{1}}^{+\operatorname{ex}}\left(\mathbf{p}_{1}\right) \ldots b_{n_{k}, \nu_{k}}^{+\operatorname{ex}}\left(\mathbf{p}_{k}\right) \Phi_{0}$ is an improper eigenvector of $H$ to the eigenvalue

To show this we write

$$
\frac{\mathbf{p}_{1}^{2}}{2 M_{1}}+E_{v_{1}}+\cdots+\frac{\mathbf{p}_{k}^{2}}{2 M_{k}}+E_{v_{k}} .
$$

$$
\begin{aligned}
b_{n \alpha \nu}^{+\mathrm{ex}}=\lim _{t \rightarrow \pm \infty} b_{n \alpha \nu}^{+}(t-\tau) & =\lim _{t \rightarrow \pm \infty} e^{-i H \tau} \underline{b}_{n \alpha \nu, \tau}^{+}(t) e^{i H \tau} \\
& =e^{-i H \tau} \underline{b}_{n \alpha \nu, \tau}^{+\mathrm{ex}} e^{i H \tau}
\end{aligned}
$$

where $\underline{b}_{n \alpha v, \tau}^{+}(t)$ is given by (3.5) and (3.6) with the test function $f_{\alpha}(\mathbf{p})$ replaced by

$$
\tilde{f}_{\alpha, \tau}(\mathbf{p})=e^{i\left(\frac{\mathbf{p}^{2}}{2 M}+E_{\nu}\right) \tau} f_{\alpha}(\mathbf{p}) .
$$

Eqs. (7.8) and (7.6) yield

$$
e^{i H \tau} b_{n \alpha \nu}^{+ \text {ex }} e^{-i H \tau}=\underline{b}_{n \alpha \nu, \tau}^{+\mathrm{ex}}=\int d^{3} \mathbf{p} \tilde{f}_{\alpha, \tau}(\mathbf{p}) b_{n, \nu}^{+\mathrm{ex}}(\mathbf{p}) .
$$

First differentiating this expression with respect to $\tau$ and then setting $\tau=0$ we get

$$
\left[H, b_{n \alpha \nu}^{+e x}\right]=\int d^{3} \mathbf{p}\left(\frac{\mathbf{p}^{2}}{2 M}+E_{v}\right) \tilde{f}_{\alpha}(\mathbf{p}) b_{n, v}^{+\mathrm{ex}}(\mathbf{p}) .
$$

From this the statement follows at once.

Acknowledgments. I would like to thank Prof. G. GRAwert for clarifying discussions and comments, Prof. H. Ekstein and Prof. D. KastLer for encouraging interest and valuable suggestions. Furthermore I am grateful to Dr. K. HePP for stimulations and to Dr. J. Kupsch for helpful discussions.

${ }^{10}$ That is, $b_{n \alpha \nu}^{+}(t)$, considered as operator-valued function of $f_{\alpha}(\mathbf{x}, 0) \in L^{2}$, depends continuously on $f_{\alpha}$. 


\section{Appendix}

\section{A. The Functions $g_{v}\left(\mathbf{x}-\mathbf{x}_{1}, \ldots, \mathbf{x}-\mathbf{x}_{n}\right)$}

The conditions I. $-I V$. given in Sec. 3 fix the functions $g_{v}\left(\mathbf{x}-\mathbf{x}_{1}, \ldots\right.$, $\mathbf{x}-\mathbf{x}_{n}$ ) introduced by Eq. (3.1). For their calculation it is convenient to use Jacobian coordinates (see, e.g., [13]):

$$
\begin{aligned}
& \xi_{i}=\frac{\left(\mathbf{x}-\mathbf{x}_{1}\right)+\cdots+\left(\mathbf{x}-\mathbf{x}_{i}\right)}{i}-\left(\mathbf{x}-\mathbf{x}_{i+1}\right)=\mathbf{x}_{i+1}-\frac{\mathbf{x}_{1}+\cdots+\mathbf{x}_{i}}{i} \\
& (i=1, \ldots, n-1) \\
& \xi_{n}=\frac{\left(\mathbf{x}-\mathbf{x}_{1}\right)+\cdots+\left(\mathbf{x}-\mathbf{x}_{n}\right)}{n}=\mathbf{x}-\frac{\mathbf{x}_{1}+\cdots+\mathbf{x}_{n}}{n}=\mathbf{x}-\mathbf{r}_{n} .
\end{aligned}
$$

Then Eq. (3.2) implies

$$
\begin{aligned}
g_{v}^{*}\left(\mathbf{x}-\mathbf{x}_{1}, \ldots, \mathbf{x}-\mathbf{x}_{n}\right)=\hat{g}_{v}\left(\mathbf{x}-\mathbf{r}_{n}, \boldsymbol{\xi}_{1}, \ldots, \boldsymbol{\xi}_{n-1}\right) \\
=\delta\left(\mathbf{x}-\mathbf{r}_{n}\right) h_{v}\left(\boldsymbol{\xi}_{1}, \ldots, \boldsymbol{\xi}_{n-1}\right)
\end{aligned}
$$

where, due to condition I, we have

$$
\left.h_{v}\left(\boldsymbol{\xi}_{1}, \ldots, \boldsymbol{\xi}_{n-1}\right) \in L^{2}\left(R^{3 n-3}\right) \quad \text { (with respect to } \boldsymbol{\xi}_{1}, \ldots, \boldsymbol{\xi}_{n-1}\right) \text {. }
$$

From (3.12), which is equivalent to (3.10) and (3.4), respectively, one now obtains

with

$$
\left[-\sum_{i=1}^{n-1} \frac{1}{2 \mu_{i}} \nabla_{\xi_{i}}^{2}+\sum_{i<j} V\left(\varrho_{i j}\right)-E_{\nu}\right] h_{v}\left(\xi_{1}, \ldots, \xi_{n-1}\right)=0
$$

and

$$
\frac{1}{\mu_{i}}=\frac{1}{i \cdot m}+\frac{1}{m}
$$

$\boldsymbol{\varrho}_{j i}=\mathrm{x}_{j}-\mathbf{x}_{i}=\xi_{j-1}-\frac{i-1}{i} \xi_{i-1}+\sum_{k=i}^{j-2} \frac{1}{k+1} \xi_{k}$, for $j>i$.

Thus $h_{\nu}\left(\boldsymbol{\xi}_{1}, \ldots, \boldsymbol{\xi}_{n-1}\right)$ is an eigenfunction of the $n$-particle Schrödinger equation with separated center-of-mass motion.

Eq. (A.2) yields at once the property (5.11). Substituting (A.2) into (5.10) we have, for $n \neq 1$,

$$
\begin{aligned}
G\left(\mathbf{x}^{\prime}, \mathbf{x}\right)=\delta\left(\mathbf{x}^{\prime}-\mathbf{x}\right) & \int d^{3} \mathbf{x}_{2} \ldots \int d^{3} \mathbf{x}_{n} \times \\
& \times \delta\left(\mathbf{x}-\frac{\mathbf{x}_{\mathbf{2}}+\cdots+\mathbf{x}_{n}}{n}\right)\left|h_{v}\left(\mathbf{x}_{2}, \boldsymbol{\xi}_{2}^{\prime}, \ldots, \boldsymbol{\xi}_{n-1}^{\prime}\right)\right|^{2}
\end{aligned}
$$

where the variables $\xi_{i}^{\prime}$ are defined by (A.1) setting there $\mathbf{x}_{1}=0$. We now put

$$
h_{v}\left(\mathbf{x}_{2}, \boldsymbol{\xi}_{2}^{\prime}, \ldots, \boldsymbol{\xi}_{n-1}^{\prime}\right)=\hat{h}_{v}\left(\frac{\mathbf{x}_{2}+\cdots+\mathbf{x}_{n}}{n}, \boldsymbol{\xi}_{2}^{\prime}, \ldots, \boldsymbol{\xi}_{n-1}^{\prime}\right)
$$

to get lastly

$$
\begin{aligned}
G\left(\mathbf{x}^{\prime}, \mathbf{x}\right) & =\delta\left(\mathbf{x}^{\prime}-\mathbf{x}\right) \int d^{3} \xi_{2}^{\prime} \ldots d^{3} \xi_{n-1}^{\prime}\left|\hat{h}_{p}\left(\mathbf{x}, \xi_{2}^{\prime}, \ldots, \xi_{n-1}^{\prime}\right)\right|^{2} \\
& =\delta\left(\mathbf{x}^{\prime}-\mathbf{x}\right) G(\mathbf{x}) .
\end{aligned}
$$


From $h_{\nu} \in L^{2}$ it follows $\hat{h}_{v} \in L^{2}$ (with respect to all its variables), and therefore $G(\mathbf{x})$ is an integrable function of $\mathbf{x}$.

We mention that in the special case $n=1$ Eq. (A.2) gives

$$
B_{n=1}(\mathbf{x}, t)=\int d^{3} \mathbf{x}_{1} \delta\left(\mathbf{x}-\mathbf{x}_{1}\right) \psi\left(\mathbf{x}_{1}, t\right)=\psi(\mathbf{x}, t) .
$$

Finally it should be stressed that we could have also started by first defining the functions $g_{v}\left(\mathbf{x}-\mathbf{x}_{1}, \ldots, \mathbf{x}-\mathbf{x}_{n}\right)$ by (A.2)-(A.4). Afterwards constructing $n$-particle operators $b_{n \alpha \nu}^{+}(t)$ by (3.1) and (3.6) the property (3.10) could have been proved. However, the method used in Sec. 3 shows more transparently the general points important for the construction of scattering states. Furthermore, this procedure is closely related to the one employed in the relativistic case [1], [4], [5], where it is impossible to start with an analogue of Eq. (3.12), while condition (3.10) can still be used [4].

\section{B. Proof of (5.21)}

In this appendix the validity of (5.21) is shown under the assumption (5.20) for $V(\mathbf{r})$. We start with the estimate (5.12) abbreviating its righthand side by $J(t)$. We now split up the integral $J(t)$ into three parts: $J(t)=J_{1}(t)+J_{2}(t)+J_{3}(t)$ such that

$$
\begin{aligned}
0 & \leqq r \leqq r_{1} \quad \text { in } J_{1} \\
r_{1} & \leqq r \leqq \frac{|t|}{t_{0}} r_{1} \quad \text { in } J_{2} \\
\frac{|t|}{t_{0}} r_{1} & \leqq r<\infty \quad \text { in } J_{3}
\end{aligned}
$$

with $|t| \geqq t_{0}>0$. Therefore it follows, with the help of (3.14),

$J_{1} \leqq K^{(n, j)} \frac{c_{\alpha}^{2}}{|t|^{3}} \int_{r=0}^{r} \int_{0}^{=r_{1}} d^{3} \mathbf{r} V^{2}(\mathbf{r}) \int d^{3} \mathbf{x} G(\mathbf{x})\left\|\Phi_{F}^{(j)}\right\|^{2} \leqq \frac{\text { const }}{|t|^{3}}\left\|\Phi_{F}^{(j)}\right\|^{2}$

From (5.20) one sees, that in the integral $J_{2}$ the estimate

$$
|V(\mathbf{r})| \leqq \frac{c}{r^{1+\beta}}, \quad \beta>0
$$

can be introduced. Using again (3.14) we get, in the case $\beta \neq 1 / 2$,

$$
\begin{aligned}
& J_{2} \leqq K^{(n, j)} \frac{c_{\alpha}^{2}}{|t|^{3}} c^{2} \frac{4 \pi}{1-2 \beta}\left[\frac{t_{0}^{2 \beta-1}}{\left(r_{1}|t|\right)^{2 \beta-1}}-\frac{1}{r_{1}^{2 \beta-1}}\right] \int d^{3} \mathrm{x} G(\mathbf{x})\left\|\Phi_{F}^{(j)}\right\|^{2} \leqq \\
& \leqq \text { const }\left(\frac{1}{|t|^{2+2} \bar{\beta}}+\frac{1}{|t|^{3}}\right)\left\|\Phi_{F}^{(j)}\right\|^{2} \text {. }
\end{aligned}
$$

For $\beta=1 / 2$ the estimate

$$
|V(\mathbf{r})| \leqq \frac{c}{r^{3 / 2}} \leqq \frac{c}{r^{3 / 2}}\left(\frac{r}{r_{1}}\right)^{\varepsilon}, \quad \varepsilon>0
$$


implies at once

In $J_{3}$ we have

$$
J_{2} \leqq \frac{c^{\prime}}{|t|^{3-2 \varepsilon}}\left\|\Phi_{F}^{(i)}\right\|^{2}, \quad c^{\prime}=c^{\prime}(\varepsilon)
$$

and therefore

$$
|V(\mathbf{r})| \leqq \frac{c}{r^{1+\beta}} \leqq c\left(\frac{t_{0}}{r_{1}|t|}\right)^{1+\beta}
$$

where

$$
\leqq \frac{\text { const }}{|t|^{2+2 \beta}}\left\|\Phi_{F}^{(j)}\right\|^{2}
$$

has been used.

Thus from (B.2), (B.4), (B.6) and (B.8) we conclude

$$
\left\|\dot{b}_{n \alpha \nu}^{+}(t, 0) \Phi^{(j)}\right\| \leqq \frac{c_{\alpha, j}}{|t|^{1+\gamma}}\left\|\Phi^{(j)}\right\|
$$

with

and

$$
\gamma=\beta \quad \text { for } \quad \beta<1 / 2, \quad \gamma=1 / 2-\varepsilon \text { for } \beta=1 / 2
$$

$$
\gamma=1 / 2 \text { for } \beta>1 / 2 \text {. }
$$

The same estimate holds, of course, if $\dot{b}_{n \alpha \nu}^{+}(t, 0)$ is replaced by $\dot{b}_{n \alpha \nu}^{+}(t)$ Q.E.D.

\section{Alternative proof of existence}

In the following we show directly the convergence of the states (4.1) not proving first the existence of $b_{n \alpha \nu}^{+ \text {ex }}$, as it was done in Sec. 5. Thi procedure is somewhat more related to the one used in the relativisti case [4], [5]. Furthermore it allows to weaken the assumptions on th potential slightly (see C.15).

Analogously to (5.3) a sufficient condition for the convergence o $(4.1)$ is :

$\left\|\frac{d}{d t} \Phi_{\eta}(t)\right\|=\left\|\frac{d}{d t} \Phi_{n_{1} \alpha_{1} v_{1}, \ldots, n_{k} \alpha_{k} v_{k}}(t)\right\| \leqq \frac{\text { const }}{|t|^{1+\gamma}}, \quad$ with $\quad \gamma>0$.

Because of

$\left\|\frac{d}{d t} \Phi_{\eta}(t)\right\| \leqq \sum_{i=1}^{k}\left\|b_{n_{1} \alpha_{1} \nu_{1}}^{+}(t) \ldots \dot{b}_{n_{i} \alpha_{i} \nu_{i}}^{+}(t) \ldots b_{n_{k} \alpha_{k} \nu_{k}}^{+}(t) \Phi_{0}\right\|=\sum_{i=1}^{k} I_{i}(t)$

we have to consider terms of the form

$$
I_{i}(t)=\left\|b_{n_{1} \alpha_{1} \nu_{1}}^{+}(t, 0) \ldots \dot{b}_{n_{i} \alpha_{i} \nu_{i}}^{+}(t, 0) \ldots b_{n_{k} \alpha_{k} \nu_{k}}^{+}(t, 0) \Phi_{0}\right\|
$$

where the definitions (5.6) and (5.16) have been used. 
Now, with the help of (3.13), one obtains

with

$$
\left[\dot{b}_{n_{i} \alpha_{i} v_{i}}^{+}(t, 0), b_{n_{j} \alpha_{j} v_{j}}^{+}(t, 0)\right]_{\mp}=\hat{b}_{i j}^{+}(t, 0)
$$

$\hat{b}_{i j}^{+}(t, 0)=\int d^{3} \mathbf{x}_{1}^{\prime} \ldots d^{3} \mathbf{x}_{n_{i}}^{\prime} \int d^{3} \mathbf{x}_{1}^{\prime \prime} \ldots d^{3} \mathbf{x}_{n_{j}}^{\prime \prime} \times$

$\times w\left(\mathbf{x}_{1}^{\prime}, \ldots, \mathbf{x}_{n_{i}}^{\prime} ; \mathbf{x}_{1}^{\prime \prime}, \ldots, \mathbf{x}_{n_{j}}^{\prime \prime}\right) \psi^{+}\left(\mathbf{x}_{1}^{\prime}\right) \ldots \psi^{+}\left(\mathbf{x}_{n_{i}}^{\prime}\right) \psi^{+}\left(\mathbf{x}_{1}^{\prime \prime}\right) \ldots \psi^{+}\left(\mathbf{x}_{n_{j}}^{\prime \prime}\right)$

and

$\left|w\left(\mathbf{x}_{1}^{\prime}, \ldots, \mathbf{x}_{n_{i}}^{\prime} ; \mathbf{x}_{1}^{\prime \prime}, \ldots, \mathbf{x}_{n_{j}}^{\prime \prime}\right)\right|=$ const $\mid \int d^{3} \mathbf{x}_{i} \int d^{3} \mathbf{x}_{j} f_{\alpha_{i}}\left(\mathbf{x}_{i}, t\right) f_{\alpha_{j}}\left(\mathbf{x}_{j}, t\right) \times$ $\times g_{v_{i}}^{*}\left(\mathbf{x}_{i}-\mathbf{x}_{1}^{\prime}, \ldots, \mathbf{x}_{i}-\mathbf{x}_{n_{i}}^{\prime}\right) V\left(\mathbf{x}_{1}^{\prime}-\mathbf{x}_{1}^{\prime \prime}\right) g_{v_{j}}^{*}\left(\mathbf{x}_{j}-\mathbf{x}_{1}^{\prime \prime}, \ldots, \mathbf{x}_{j}-\mathbf{x}_{n_{j}}^{\prime \prime}\right) \mid$.

To simplify the notation we write

and thus it follows

$$
b_{n_{i} \alpha_{i} \nu_{i}}^{+}(t, 0)=b_{i}^{+}
$$

$$
I_{i}(t) \leqq \sum_{j=i+1}^{k}\left\|b_{1}^{+} \ldots b_{i-1}^{+} b_{i+1}^{+} \ldots b_{j-1}^{+} b_{j+1}^{+} \ldots b_{k}^{+} \hat{b}_{i j}^{+}(t, 0) \Phi_{0}\right\| .
$$

Here we have used, besides (C.4), that $\hat{b}_{i j}^{+}(t, 0)$ (anti-)commutes with $b_{n \alpha \nu}^{+}(t, 0)$. As it was shown in Sec. 5 the $b_{i}^{+}$are bounded operators (see Eq. (5.17)), i.e.,

$$
I_{i}(t) \leqq \text { const } \cdot \sum_{j=i+1}^{k}\left\|\hat{b}_{i j}^{+}(t, 0) \Phi_{0}\right\|
$$

holds. With the help of (5.8) we find

$$
\begin{gathered}
\left\|\hat{b}_{i j}^{+}(t, 0) \Phi_{0}\right\|^{2} \leqq \text { const } \int d^{3} \mathbf{x}_{1}^{\prime} \ldots d^{3} \mathbf{x}_{n_{i}}^{\prime} \int d^{3} \mathbf{x}_{1}^{\prime \prime} \ldots d^{3} \mathbf{x}_{n_{j}}^{\prime \prime}\left|w\left(\mathbf{x}_{1}^{\prime}, \ldots, \mathbf{x}_{n_{i}}^{\prime} ; \mathbf{x}_{1}^{\prime \prime}, \ldots, \mathbf{x}_{n_{j}}^{\prime \prime}\right)\right|^{2} \\
=\mathrm{const} \int d^{3} \mathbf{y}_{i} \int d^{3} \mathbf{y}_{j} \int d^{3} \mathbf{x}_{i} \int d^{3} \mathbf{x}_{j} \int d^{3} \mathbf{r} \times \\
\times f_{\alpha_{i}}^{*}\left(\mathbf{y}_{i}, t\right) f_{\alpha_{i}}\left(\mathbf{x}_{i}, t\right) f_{\alpha_{j}}^{*}\left(\mathbf{y}_{j}+\mathbf{r}, t\right) f_{\alpha_{j}}\left(\mathbf{x}_{j}+\mathbf{r}, t\right) \times \\
\times V^{2}(\mathbf{r}) G_{i j}\left(\mathbf{y}_{i}-\mathbf{x}_{i}, \mathbf{y}_{j}-\mathbf{x}_{j}, \mathbf{x}_{i}-\mathbf{x}_{j}\right)
\end{gathered}
$$

where $\mathbf{x}_{1}^{\prime \prime}-\mathbf{x}_{1}^{\prime}=\mathbf{r}$ has been introduced and

$$
\begin{aligned}
& G_{i j}\left(\mathbf{y}_{i}-\mathbf{x}_{i}, \mathbf{y}_{j}-\mathbf{x}_{j}, \mathbf{x}_{i}-\mathbf{x}_{j}\right)=\int d^{3} \mathbf{z} \int d^{3} \mathbf{x}_{2}^{\prime} \ldots d^{3} \mathbf{x}_{n_{i}}^{\prime} \int d^{3} \mathbf{x}_{2}^{\prime \prime} \ldots d^{3} \mathbf{x}_{n_{j}}^{\prime \prime} \times \\
& \times g_{v_{i}}\left(\mathbf{y}_{i}-\mathbf{z}, \mathbf{y}_{i}-\mathbf{x}_{2}^{\prime}, \ldots, \mathbf{y}_{i}-\mathbf{x}_{n_{i}}^{\prime}\right) g_{v_{i}}^{*}\left(\mathbf{x}_{i}-\mathbf{z}, \mathbf{x}_{i}-\mathbf{x}_{2}^{\prime}, \ldots, \mathbf{x}_{i}-\mathbf{x}_{n_{i}}^{\prime}\right) \times(\mathrm{C} .11) \\
& \times g_{v_{j}}\left(\mathbf{y}_{j}-\mathbf{z}, \mathbf{y}_{j}-\mathbf{x}_{2}^{\prime \prime}, \ldots, \mathbf{y}_{j}-\mathbf{x}_{n_{j}}^{\prime \prime}\right) g_{v_{j}}^{*}\left(\mathbf{x}_{j}-\mathbf{z}, \mathbf{x}_{j}-\mathbf{x}_{2}^{\prime \prime}, \ldots, \mathbf{x}_{j}-\mathbf{x}_{n_{j}}^{\prime \prime}\right) .
\end{aligned}
$$

Considering the specific properties of the functions $g_{v}\left(\mathbf{x}-\mathbf{x}_{1}, \ldots, \mathbf{x}-\mathbf{x}_{n}\right)$ given in (A.2) we obtain

$G_{i j}\left(\mathbf{y}_{i}-\mathbf{x}_{i}, \mathbf{y}_{j}-\mathbf{x}_{j}, \mathbf{x}_{i}-\mathbf{x}_{j}\right)=\delta\left(\mathbf{y}_{i}-\mathbf{x}_{i}\right) \delta\left(\mathbf{y}_{j}-\mathbf{x}_{j}\right) \hat{G}_{i j}\left(\mathbf{x}_{i}-\mathbf{x}_{j}\right)$

where the $\hat{G}_{i j}\left(\mathbf{x}_{i}-\mathbf{x}_{j}\right)$ are $\delta$-functions or, as a consequence of condition I, integrable functions (compare the corresponding property (5.11)).

Therefore the estimate

$$
\begin{aligned}
\left\|\hat{b}_{i j}^{+}(t, 0) \Phi_{0}\right\|^{2} \leqq \text { const } \int d^{3} \mathbf{x}_{i} \int d^{3} \mathbf{x}_{j} \int d^{3} \mathbf{r} \times \\
\times\left|f_{\alpha_{i}}\left(\mathbf{x}_{i}, t\right)\right|^{2}\left|f_{\alpha_{j}}\left(\mathbf{x}_{j}+\mathbf{r}, t\right)\right|^{2} V^{2}(\mathbf{r}) \hat{G}_{i j}\left(\mathbf{x}_{i}-\mathbf{x}_{j}\right)
\end{aligned}
$$


follows and we find, if (5.13) or (5.20) is fulfilled (cf. Appendix B):

$$
\left\|\hat{b}_{i j}^{+}(t, 0) \Phi_{0}\right\|^{2} \leqq \frac{\text { const }}{|t|^{2+2 \gamma}} \quad, \gamma>0 \text {. Q.E.D. }
$$

The validity of (C.1) can also be shown under the following somewhat less restrictive assumption for the potential [14]:

There exists an $\varepsilon>0$ such that

$$
\int(r+1)^{\varepsilon-1} V^{2}(\mathbf{r}) d^{3} \mathbf{r}<\infty
$$

(without loss of generality we take $\varepsilon \leqq 1$ ).

Using instead of (3.14) the estimate [15]

$$
(r+1)^{\beta}\left|f_{\alpha}(\mathbf{r}, t)\right| \leqq c_{\alpha, \beta}|t|^{\beta-3 / 2},
$$

valid for any $\beta \geqq 0$, we obtain by virtue of (C.13)

$$
\left\|\hat{b}_{i j}^{+}(t, 0) \Phi_{0}\right\| \leqq \frac{\text { const }}{|t|^{1+\varepsilon / 2}} \text {. Q.E.D. }
$$

We note that for the proof of this inequality the strong decrease of the bound-state functions (A.2) and therefore of $\hat{G}_{i j}(\mathbf{x})$ (for large values of its variables) is important. It already suffices if $\int d^{3} \mathbf{x}|\mathbf{x}|^{1-\varepsilon} \hat{G}_{i j}(\mathbf{x})$ exists, which is fulfilled, if the same property holds for $\left|h_{\nu}\left(\xi_{1}, \ldots, \xi_{n-1}\right)\right|^{2}$ with respect to each of its variables.

\section{References}

[1] Ekstein, H.: Phys. Rev. 101, 880 (1956); Nuovo cimento 4, 1017 (1956).

[2] J $\mathrm{AUCH}$, J. M.: Helv. Phys. Acta 31, 661 (1958). - HACK, M. N.: Nuovo cimento 13, 231 (1959).

[3] BrentG, W., and R. HAaG: Fortschr. Phys. 7, 183 (1959).

[4] HAAG, R.: Phys. Rev. 112, 669 (1958).

[5] Ruelle, D.: Helv. Phys. Acta 35, 147 (1962).

[6] Cook, J. M.: J. Math. and Phys. 36, 82 (1957). - HACK, M. N.: Nuovo cimento 13, 231 (1959).

[7] HACK, M. N.: Nuovo cimento 9, 731 (1958).

[8] SchweBer, S. S.: An Introduction to Relativistic Quantum Field Theory. Evanston, Ill.: Row, Peterson and Co. 1961.

[9] Ekstein, H.: Nuovo cimento 4, 1017 (1956). - Redmond, P. J., and J. L. Uretsky: Ann. Phys. 9, 106 (1960). - Tani, S.: Phys. Rev. 117, 252 (1960); 121, 346 (1961). - Schweber, S. S., and E. C. G. SudarshaN: Ann. Phys. 19, 351 (1962). - WEIDLICH, W.: Z. Naturforsch. 18a, 1266 (1963). - Stichel, P.: Nuclear Physics 52, 189 (1964).

[10] Kato, T.: Trans. Am. Math. Soc. 70, 195 (1951).

[11] Schwartz, L.: Théorie des distributions. Vol. II, p. 89 ff. Paris: Hermann 1959.

[12] Dollard, J. D.: J. Math. Phys. 5, 729 (1964).

[13] Blochinzew, D. I.: Grundlagen der Quantenmechanik, p. $372 \mathrm{ff}$. and p. $532 \mathrm{f}$. Berlin: Deutscher Verlag der Wissenschaften 1953.

[14] Kuroda, S. T.: Nuovo cimento 12, 431 (1959).

[15] Kupsch, J., and W. Sandhas: Commun. Math. Phys. 2, 147 (1966). 